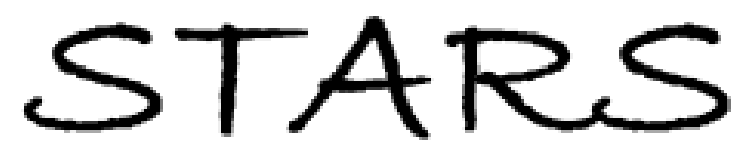

University of Central Florida

STARS

1-1-1994

\title{
A Model Cylindrical Magnetron Vlasov Distribution Function
}

D. J. Kaup

S. Roy Choudhury

University of Central Florida

Find similar works at: https://stars.library.ucf.edu/facultybib1990

University of Central Florida Libraries http://library.ucf.edu

This Article is brought to you for free and open access by the Faculty Bibliography at STARS. It has been accepted for inclusion in Faculty Bibliography 1990s by an authorized administrator of STARS. For more information, please contactSTARS@ucf.edu.

\section{Recommended Citation}

Kaup, D. J. and Choudhury, S. Roy, "A Model Cylindrical Magnetron Vlasov Distribution Function" (1994). Faculty Bibliography 1990s. 1086.

https://stars.library.ucf.edu/facultybib1990/1086

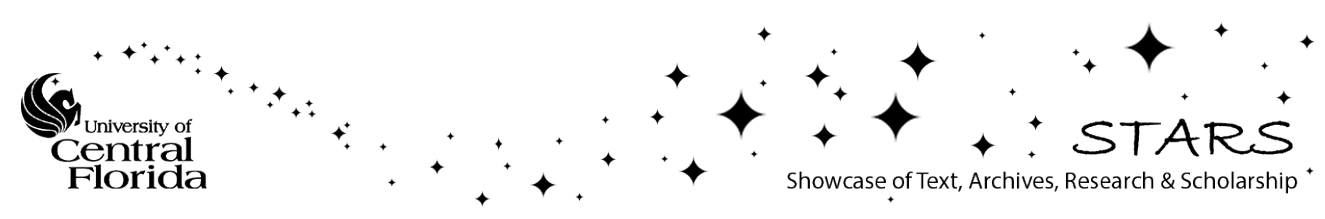




\section{A model cylindrical magnetron Vlasov distribution function}

Cite as: Physics of Plasmas 1, 3437 (1994); https://doi.org/10.1063/1.870492

Submitted: 28 February 1994 . Accepted: 10 June 1994 . Published Online: 04 June 1998

D. J. Kaup, and S. Roy Choudhury

\section{ARTICLES YOU MAY BE INTERESTED IN}

Magnetic insulation at finite temperatures

Physics of Plasmas 13, 083104 (2006); https://doi.org/10.1063/1.2244529

Limiting current in a relativistic diode under the condition of magnetic insulation

Physics of Plasmas 10, 4489 (2003); https://doi.org/10.1063/1.1613654

A model magnetron distribution function: Analysis and scalings

The Physics of Fluids 31, 2362 (1988); https://doi.org/10.1063/1.866584

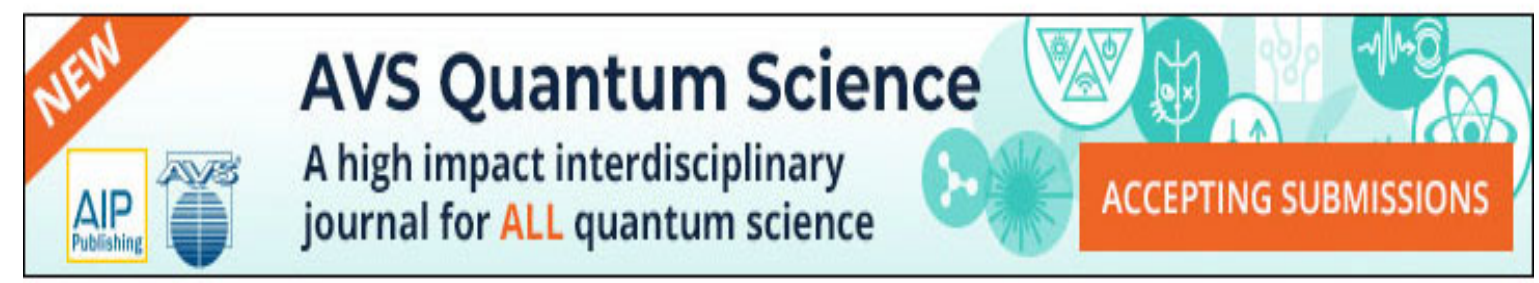




\title{
A model cylindrical magnetron Vlasov distribution function
}

\author{
D. J. Kaup \\ Department of Mathematics and Computer Science, Clarkson University, Potsdam, New York 13699-5815 \\ S. Roy Choudhury \\ Department of Mathematics, University of Central Florida, Orlando, Florida 32816
}

(Received 28 February 1994; accepted 10 June 1994)

The analysis of the planar magnetron Vlasov distribution function [Phys. Fluids 31, 2362 (1988)] is extended to the cylindrical case. In momentum space, the model distribution function is $f\left(w, p_{\theta}\right)=\mathrm{Ne}^{-\beta_{w} w} e^{-\left(\Omega \beta_{\theta} / 4 p_{0}\right)\left(p_{\theta}-p_{0}\right)^{2}}$ where $w\left(p_{\theta}\right)$ is the single particle energy (angular momentum), $\beta_{u}\left(\beta_{\theta}\right)$ is the inverse of the thermal energy associated with variations in $w\left(p_{\theta}\right), p_{0}$ is the angular momentum at the cathode, and $\Omega$ is the electron cyclotron frequency $\left(=e B_{0} / m c\right)$. The problem is shown to be too "stiff" numerically to permit a pure numerical solution even using very high accuracy and state-of-the-art numerical schemes. It is shown that one may use a global singular perturbation expansion, similar to, but significantly more complex than the one used in the planar case, to solve the resulting nonlinear ordinary differential equation for the spatial dependence of the distribution function, density, electrostatic potential, and drift velocity.

\section{INTRODUCTION}

Models of magnetrons and crossed-field amplifiers (CFA's) in planar geometry have existed for some time. ${ }^{1,2}$ This geometry is quite convenient mathematically for studying such devices. ${ }^{3,4}$ Planar models have also been used in numerical simulations. ${ }^{5,6}$ Cylindrical devices have also been studied theoretically in the cold-fluid $\operatorname{limit}^{7}$ as well as numerically with particle simulations. However, there has existed no adequate Vlasov model in cylindrical geometry up to the present time. The planar geometry is quite adequate for low aspect ratio devices such as most CFA's. (The aspect ratio is the ratio of the anode radius to the cathode radius.) However, magnetrons typically have high aspect ratios wherein cylindrical deviations from planar geometry can be expected to be quite important. In this paper, a model Vlasov distribution function is provided for a cylindrical magnetron. In many ways, it is quite similar to the planar model, however, there are important geometrical deviations. Still, the basic governing function is exactly the same function $J_{0}(z)$ defined in the planar model. ${ }^{2}$

In Sec. II, the model is described and is transformed to velocity space. In Sec. III, it is shown that one may use a singular perturbation expansion to solve the nonlinear Poisson's equation. In Sec. IV, the results for typical medium and high aspect ratio devices are presented. Here we discuss and demonstrate the high degree of stretching which occurs in this problem and describe how one may determine all the parameters required for the solution via the asymptotic expansion. Then we present curves of the density profiles for the medium and high aspect ratio cases. In general, the curves are as one would expect, with high densities in the interiors and a rather sharp and well-defined edge. Last, we discuss the stability of the partical orbits, concluding that they are indeed stable. However, small perturbations can cause very large excursions.

\section{THE CYLINDRICAL MODEL}

We shall use the geometry detailed in Fig. 1 where $a(b)$ is the cathode (anode) radius, the B field (assumed uniform) is directed into the paper and the anode is at some positive potential, creating an electric field from the anode to the cathode. Here we are only interested in the stationary characteristics so we assume azimuthal symmetry, with all physical quantities independent of the azimuthal angle, $\theta$.

The form of the electrostatic field and potential will follow from Poisson's equation,

$$
\left[\partial_{r}^{2}+(1 / r) \partial_{r}\right] \phi=4 \pi e n,
$$

where $n$ is the azimuthally symmetric electron density. 'The anode-cathode voltage will pull electrons out of the cathode, creating a sheath next to the cathode. This sheath is magnetically insulated from the anode by the magnetic field, $B_{0}$, and will grow until we have a space-charge limited current at the cathode, with a vanishing $E$ field at the cathode. Taking the potential to be zero at the cathode, then our boundary conditions on (1) at the cathode are

$$
\phi(r=a)=0=\partial_{r} \phi(r=a) .
$$

This will uniquely determine $\phi(r)$ from (1) once the electron density is determined.

The electron density will be determined by a model Vlasov distribution function. The model that we will use will be

$$
f\left(w, p_{\theta}\right)=\mathrm{Ne}^{-\beta_{w} w} e^{-\left(\Omega \beta_{\theta} / 4 p_{0}\right)\left(p_{\theta}-p_{0}\right)^{2}} .
$$

In the above, $N$ is a normalization constant to be determined (it will be a function of the anode voltage). The next term is the standard Gibbs term for the distribution of single-particle energies, $w$, where $\beta_{w}$ is the inverse of thermal energy. The last term arises because all electrons leave the cathode at near zero velocity. The coefficient $\beta_{\theta}$ determines the spread in these velocities about zero velocity, $p_{\theta}$ is the single par- 


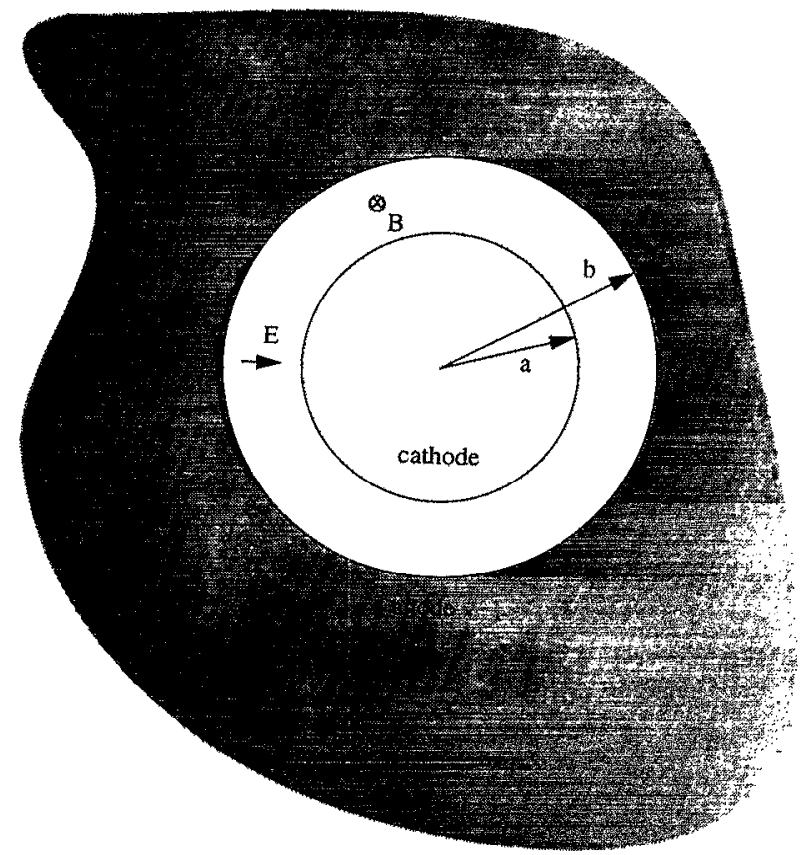

FIG. 1. The geometry for a smooth bore magnetron. The inner radius is $a$ and the outer radius is $b$.

ticle angular momentum (a constant of the motion) and $p_{0}$ is its value at the cathode. The quantity $\Omega$, which is the electron cyclotron frequency $\left[=e B_{0} /(m c)\right]$, is there simply to normalize $\beta_{\theta}$ to an inverse energy, and the factor of 4 is simply a convenient scale factor. Since both $w$ and $p_{\theta}$ are constants of the single particle motion, this $f\left(w, p_{\theta}\right)$ automatically satisfies the Vlasov equation

$$
\partial_{t} f+\mathbf{v} \cdot \nabla f-\frac{e}{m}\left[\mathbf{E}+(1 / c) \mathbf{v} \times \mathbf{B}_{0}\right] \cdot \nabla_{v} f=0
$$

where

$$
\mathbf{E}=-\nabla \phi \text {. }
$$

In cylindrical coordinates, ignoring variations in the $z$ direction, the single particle equations in the presence of an electric field and a magnetic field are

$$
\begin{aligned}
& \ddot{r}-r \dot{\theta}^{2}=(e / m) \partial_{r} \phi+\Omega r \dot{\theta}, \\
& r \ddot{\theta}+2 \dot{r} \dot{\theta}=-\Omega \dot{r}
\end{aligned}
$$

when $\partial_{\theta} \phi=0$. These equations have the two constants of the motion:

$$
\begin{aligned}
& w=(m / 2)\left(v_{r}^{2}+v_{\theta}^{2}\right)-e \phi, \\
& p_{\theta}=m r v_{\theta}+\frac{1}{2} m r^{2} \Omega,
\end{aligned}
$$

where

$$
v_{r}=\dot{r}, \quad v_{\theta}=r \dot{\theta}
$$

As stated above, we define $p_{0}$ to be that value of $p_{\theta}$ at the cathode for which $v_{\theta}=0$. Thus

$$
p_{0}=\frac{1}{2} m a^{2} \Omega
$$

For a laminar flow, we have $\dot{r}=0$ and $\ddot{\theta}=0$. Then by $(6)$, there are two possible values for the drift velocity, $v_{d}=r \theta$, both of which are negative:

$$
v_{d}=-\frac{1}{2} r \Omega \pm \sqrt{\frac{1}{4} r^{2} \Omega^{2}-\frac{e}{m}\left(r \partial_{r} \phi\right)} .
$$

The upper sign corresponds to the drift velocity of the planar case. It also vanishes at the cathode, and therefore corresponds to the typical values present in our model distribution function. The lower sign has a nonvanishing value at the cathode $\left(v_{d}=-a \Omega\right)$ and for nominal values of $\beta_{\theta}$, can be ignored as being unphysical.

To calculate the electron density and the average particle velocity, we have to convert $f$ from a function of $w$ and $p_{\theta}$ to a function of $v_{r}$ and $v_{\theta}$. This is easily done with (7), (8), and (10). The result is

$$
f=N e^{\chi} e^{-(m / 2) \beta_{w} v_{r}^{2}} e^{-(m / 2)\left[\beta_{w}+\left(r^{2} / a^{2}\right) \beta_{\theta}\right]\left(v_{\theta}+u\right)^{2}},
$$

where

$$
\begin{aligned}
& \chi=\beta_{w} e \phi-h(r), \\
& u=\beta_{\theta} r\left(r^{2}-a^{2}\right) \Omega / 2\left(a^{2} \beta_{w}+r^{2} \beta_{\theta}\right) \\
& h(r)=m \Omega^{2} \beta_{w} \beta_{\theta}\left(r^{2}-a^{2}\right)^{2} / 8\left(a^{2} \beta_{w}+r^{2} \beta_{\theta}\right) .
\end{aligned}
$$

Taking the velocity averages of (12) now gives

$$
\begin{aligned}
& n=\iint f d v_{r} d v_{\theta}=2 \pi N e^{X} / \sqrt{\beta_{w}\left(\beta_{w}+r^{2} \beta_{\theta} / a^{2}\right)}, \\
& \bar{v}_{\theta}=\frac{1}{n} \iint f v_{\theta} d v_{r} d v_{\theta}=-u,
\end{aligned}
$$

showing $u$ to be the negative of the average azimuthal velocity.

Near the cathode, we expect the electron plasma frequency to be just below the electron-cyclotron frequency by some temperature factor. With this in mind, we rescale $N$ as

$$
2 \pi N=\frac{m}{4 \pi e^{2}} \Omega^{2} \beta_{\theta}\left(\frac{\beta_{w}}{\beta_{w}+\beta_{\theta}}\right)^{1 / 2}(1-\epsilon)
$$

where $\epsilon$ is now the free parameter. With this, (16) gives

$$
\omega_{p}^{2}=\frac{4 \pi e^{2}}{m} n=\frac{\beta_{\theta} \Omega^{2}(1-\epsilon) e^{\chi}}{\sqrt{\beta_{w}+\beta_{\theta}} \sqrt{\beta_{w}+\beta_{\theta} r^{2} / a^{2}}},
$$

which at the cathode is

$$
\omega_{p}^{2}(r=a)=\frac{\beta_{\theta} \Omega^{2}(1-\epsilon)}{\beta_{w}+\beta_{\theta}} .
$$

Now the only remaining equation to solve is Poisson's. From (1) we then have

$$
\left[\partial_{r}^{2}+(1 / r) \partial_{r}\right][(e / m) \phi]=\omega_{p}^{2}
$$

where $\omega_{p}^{2}$ is given by (19). This is a nonlinear ODE (ordinary differential equation), since $X$ in (19) is proportional to $\phi$ [see (13)]. This is a particularly complex ODE because of the geometrical factors of $r / a$ in (19) and (15). Attempts to solve this equation numerically have failed, even with 40 place accuracy. However, as we shall shortly see, these cylindrical 
factors actually only introduce some minor varialions from the planar case. The key point is that the factor of $e^{x}$ in $\omega_{p}^{2}$ in (21) and (19) dominates all geometrical effects. This was the key for the asymptotic expansion given in the next section.

\section{THE ASYMPTOTIC EXPANSION}

Due to secondary emission at the cathode, one expects values for $\beta_{w}$ and $\beta_{\theta}$ to be in the order of inverse electron volts. Since $m a^{2} \Omega^{2}$ is typically keV's, we have here a small parameter for expansion. And as we shall see later, $\epsilon$ in (19) is typically $10^{-8}$ or even much smaller, giving us a second small parameter.

But before we expand in these parameters, let us rescale (21) and (19). We start by rescaling $r$ as

$$
r=a e^{v}
$$

so that

$$
\partial_{r}^{2}+\frac{1}{r} \partial_{r}=\frac{e^{-2 v}}{a^{2}} \partial_{v}^{2}
$$

Thus $v$ ranges from zero to $\ln (b / a)$. We introduce the ratio of the temperatures

$$
\rho=\beta_{w^{\prime}} / \beta_{\theta}
$$

and define the large unitless parameter $L$ by

$$
L^{2}=a^{2} \Omega^{2} m \beta_{w} /(1+\rho) .
$$

Then (15) and (21) become

$$
\begin{aligned}
& h=\left(L^{2} / 8\right)\left[(1+\rho) /\left(e^{2 v}+\rho\right)\right]\left(e^{2 v}-1\right)^{2}, \\
& \omega_{p}^{2}=\left[L^{2}(1-\epsilon) / a^{2} \beta_{w} m\right] e^{\chi} \mu,
\end{aligned}
$$

where

$$
\mu \equiv\left[(1+\rho) /\left(e^{2 v}+\rho\right)\right]^{1 / 2} .
$$

Define the scaled Laplacian of $h$ to be

$$
H \equiv\left(a^{2} / L^{2}\right)\left[\partial_{r}^{2}+(1 / r) \partial_{r}\right] h
$$

which is

$$
\begin{aligned}
H= & \frac{(\rho+1)}{2\left(\rho+e^{2 u}\right)^{3}}\left[e^{6 v}+3 \rho e^{4 v}+e^{2 v}+\rho(1+2 \rho)\right. \\
& \left.\times\left(2 e^{2 v}-1\right)\right]
\end{aligned}
$$

and is clearly positive definite.

With the above and (13), (21) becomes

$$
e^{-2 v} L^{-2} \partial_{v}^{2} \chi+H-\mu(1-\epsilon) e^{\chi}=0
$$

For large $L$ and small $\epsilon$, the solution of this equation can be expanded in the following asymptotic series in $v$ and $z$ :

$$
\begin{aligned}
\chi= & -J(z)+F_{0}(v)+\sum_{m, n} \frac{\epsilon^{m}}{L^{n}} J_{m n}(z) F_{m n}(v) \\
& +\sum_{m, n} \frac{\epsilon^{m}}{L^{n}} K_{m n}(z) G_{m n}(v)[\epsilon \sinh (L \nu)],
\end{aligned}
$$

where the sum is from $m$ and $n=0$ to infinity and

$$
z \equiv \epsilon \cosh (L \nu)-\epsilon,
$$

where $\nu(v)$ is some function of $v$, still to be determined.

Before we start the expansion, we should remark on certain features of it, so that one can more clearly see how the various terms will balance in the various orders. First, we note that the $e^{\chi}$ term in (30) will simply generate another series like (31). However, what will happen to the cosh in (32) and its derivatives when one does the differentiation in (30), requires a detailed discussion. Let us look at each type of term, one by one. First, for $J(z)$, we have

$$
\begin{aligned}
\left(1 / L^{2}\right) \partial_{v}^{2} J(z)= & \nu_{v}^{2}\left(z^{2} J^{\prime \prime}+z J^{\prime}\right)+\epsilon \nu_{v}^{2}\left(2 z J^{\prime \prime}+J^{\prime}\right) \\
& +(1 / L) \nu_{v v}[\epsilon \sinh (L \nu)] J^{\prime}
\end{aligned}
$$

where $J^{\prime}=d J / d z$ and $\nu_{v}=d \nu / d v$. Note how this is a sum of products of functions of $z$ and functions of $v$, with higherorder terms present $\left[\epsilon, L^{-1} \epsilon \sinh (L \nu)\right]$. It is the same form as (31). Similarly,

$$
\left(1 / L^{2}\right) \partial_{v}^{2} F_{0}(v)=\left(1 / L^{2}\right) F_{0}^{\prime \prime}
$$

has only the higher-order term $\left[L^{-2}\right]$. Continuing, 
$\frac{1}{L^{2}} \partial_{v}^{2}\left(J_{m n} F_{m n}\right)=\nu_{v}^{2} F_{m n}\left(z^{2} J_{m n}^{\prime \prime}+z J_{m n}^{\prime}\right)+\epsilon \nu_{v}^{2} F_{m n}\left(2 z J_{m n}^{\prime \prime}+J_{m n}^{\prime}\right)+\frac{1}{L} J_{m n}^{\prime}\left(\nu_{v v} F_{m n}+2 \nu_{v} F_{m n}^{\prime}\right)[\epsilon \sinh (L \nu)]+\frac{1}{L^{2}} J_{m n} F_{m n}^{\prime \prime}$,

$\frac{1}{L^{2}} \partial_{v}^{2}\left[K_{m n} G_{m n} \epsilon \sinh (L \nu)\right]=\nu_{v}^{2}\left[\left(z^{2} K_{m n}^{\prime}\right)^{\prime}+\left(K_{m n} z\right)^{\prime}\right] G_{m n}[\epsilon \sinh (L \nu)]+\epsilon \nu_{v}^{2}\left[2\left(K_{m n}^{\prime} z\right)^{\prime}+K_{m n}^{\prime}\right] G_{m n}[\epsilon \sinh (L \nu)]$

$$
\begin{aligned}
& +\frac{1}{L}\left(K_{m n}^{\prime} z^{2}+K_{m n} z\right)\left(2 \nu_{v} G_{m n}^{\prime}+\nu_{v v} G_{m n}\right)+\frac{\epsilon}{L}\left(2 K_{m n}^{\prime} z+K_{m n}\right)\left(2 \nu_{v} G_{m n}^{\prime}+\nu_{v v} G_{m n}\right) \\
& +\frac{1}{L^{2}} K_{m n} G_{m n}^{\prime \prime} \epsilon \sinh (L \nu)
\end{aligned}
$$

each of which has the same structure as (31). Thus when (31) is inserted into (30), we will generate a general sum of the form of (31) consisting of the sum of products of functions of $z$ times functions of $v$. The coefficients will be of various orders of $1 / L, \epsilon$, and $\epsilon \sinh (L \nu)$, as in (31). Thus it will be possible to collect like terms.

It is now simply a matter of inserting these results into (30), collecting the various powers and solving for the various functions of $v$ and $z$. The leading order gives

$$
e^{-2 v} \nu_{v}^{2}\left(z^{2} J^{\prime \prime}+z J^{\prime}\right)+H-\mu e^{-J} e^{F_{0}}=0 .
$$

Separating the functions of $z$ and $v$ gives

$$
\begin{aligned}
& F_{0}=\ln (H / \mu), \\
& \nu_{v}^{2}=e^{2 v} H, \\
& z^{2} J^{\prime \prime}+z J^{\prime}-1+e^{-J}=0,
\end{aligned}
$$

where we have chosen the separation ratios to be unity. Since $H$ and $\mu$ are known and are positive definite, solutions exist for $F_{0}$ and $\nu_{v}$. Equation (37) is exactly the same as in the planar case and is Eq. (31) in Ref. 2. It satisfies

$$
J(0)=0, \quad J^{\prime}(0)=1 .
$$

Thus we have a solution in leading order. If we go one more order, we only need

$$
\chi=-J(z)+F_{0}(z)+\epsilon J_{10}(z) F_{10}(v)
$$

which inserted into (30) gives

$$
\begin{aligned}
& F_{10}(v)=1, \\
& z^{2} J_{10}^{\prime \prime}+z J_{10}^{t}-e^{-J} J_{10}=-e^{-J}+2 z J^{\prime \prime}+J^{\prime} .
\end{aligned}
$$

The latter is a linear second-order nonhomogeneous ODE, whose solution is

$$
J_{10}=1-(1+z / 3) J^{\prime}
$$

for $J_{10}(0)=0=J_{10}^{\prime}(0)$. The higher-order terms $\left(\epsilon^{2}\right.$, $\left.\epsilon / L, L^{-2}\right)$ will not be given here, since they will be at least two orders of magnitude smaller.

Finally, we will give expressions for $\phi, \partial_{r} \phi$, and $\omega_{p}^{2}$ based on (39). From (13), (22), (25), and (27)

$\frac{e \phi}{m a^{2} \Omega^{2}}=\frac{\left(e^{2 v}-1\right)^{2}}{8\left(e^{2 v}+\rho\right)}+\frac{1}{(1+\rho) L^{2}}\left[-J+\ln (H / \mu)+\epsilon J_{01}\right]$,

$$
\begin{aligned}
& \frac{e}{m a^{2} \Omega^{2}} a \partial_{r} \phi \\
& =e^{v} \frac{\left(e^{2 v}-1\right)\left(e^{2 v}+1+2 \rho\right)}{4\left(e^{2 v}+\rho\right)^{2}}-\frac{e^{-v} \nu_{v}}{L(1+\rho)}\left(J^{\prime}-\epsilon J_{01}^{\prime}\right) \\
& \quad \times[\epsilon \sinh (L \nu)]+\frac{e^{-v}}{L^{2}(1+\rho)}\left(\frac{H^{\prime}}{H}-\frac{\mu^{\prime}}{\mu}\right), \\
& \frac{\omega_{p}^{2}}{\Omega^{2}}=\frac{H}{1+\rho} e^{-J}\left(1-\epsilon+\epsilon J_{01}\right) .
\end{aligned}
$$

Now, in (43) and (44), although the terms involving $J$ and $J^{\prime}$ are of lower order, once $z$ becomes large (outside the sheath), $J$ does become of order $L^{2}$ and $J^{\prime}$ becomes of order $L$. So these terms do have to be retained, in general.

\section{RESULTS}

For parameters, we use those for the Varian VMS-1873 magnetron, ${ }^{8}$ where $a=1.65 \mathrm{~cm}, b=2.61 \mathrm{~cm}$, and $\Omega=5.88 \times 10^{9} \mathrm{~Hz}$, corresponding to $B_{0}=2.1 \mathrm{kG}$. We call this parameter set a medium aspect ratio case. For the thermal parameters, we can only guess as to their values. Although the values of $\beta_{\theta}=1 /(10 \mathrm{eV})$ and $\beta_{w}=\beta_{\theta} / 8 \mathrm{might}$ be on the small side, they are not unreasonable, based on other considerations. ${ }^{9}$ As a second case, we also look at a large aspect ratio device where we keep $\Omega$ and the $\beta$ 's the same, but use $a=0.125^{\prime \prime}$ and $b=2^{\prime \prime}$ instead. Equilibrium distributions are found to exist in both cases with stable (albeit large) particle orbits.

TABLE I. The $\epsilon$ values required for various anode voltages in the medium and large aspect ratio cases.

\begin{tabular}{cccc}
\hline \hline Medium aspect ratio & \multicolumn{3}{c}{ High aspect ratio } \\
\cline { 1 - 1 } Anode voltage $(\mathrm{kV})$ & $\epsilon$ & Anode voltage $(\mathrm{kV})$ & $\epsilon$ \\
\hline 25 & $7.9672 \times 10^{-4}$ & 100 & $3.2613 \times 10^{-21}$ \\
50 & $8.3877 \times 10^{-6}$ & 250 & $1.1013 \times 10^{-32}$ \\
75 & $6.3074 \times 10^{-8}$ & 350 & $3.3379 \times 10^{-45}$ \\
100 & $3.1028 \times 10^{-10}$ & & \\
150 & $1.1215 \times 10^{-15}$ & & \\
200 & $1.5812 \times 10^{-23}$ & & \\
\hline \hline
\end{tabular}




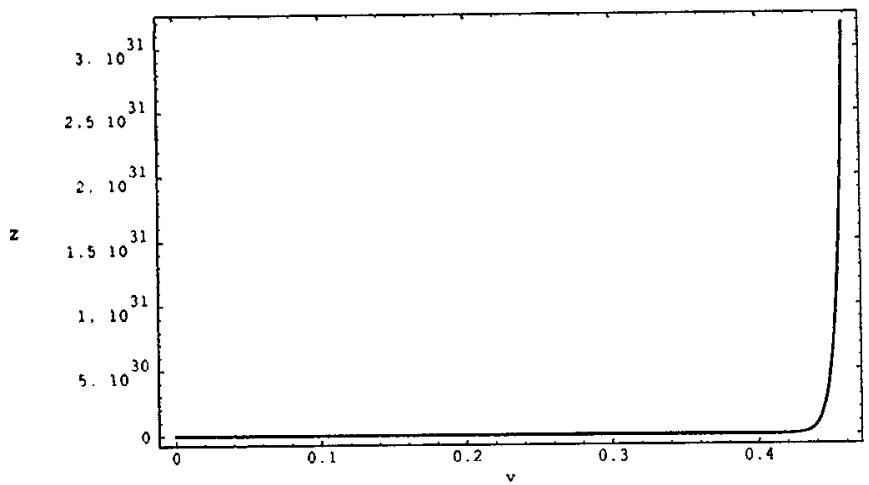

FIG. 2. Stretched $(z)$ versus physical $(v)$ variables for the medium aspect ratio case, as determined from Eqs. (32) and (36) for an anode voltage of 25 kV.

\section{A. Pure numerical solution}

Poisson's equation (21) [with (13) and (19)] can have solutions for the potential $\phi$ blowing up exponentially (due to the driving term on the right of the form $\left.e^{\chi}=e^{\beta_{w} e \phi}\right)$. Clearly, to prevent such a blowup, $\chi$ must remain close to zero, or go negative for $r \in[a, b]$. It was, therefore, deemed to be more advantageous to consider the equivalent equation for the quantity $\chi$, since this is the quantity critically affecting the nature of the solutions. Taking the second derivative of (13) and using (19) and (21) yields

$$
\frac{d^{2} \chi}{d r^{2}}=\beta_{w \cdot}\left(\frac{m \beta_{\theta} \Omega^{2}(1-\epsilon) e^{\chi}}{\sqrt{\beta_{w}+\beta_{\theta} \sqrt{\beta_{w}+\beta_{\theta} r^{2} / a^{2}}}}-\frac{1}{r} e \frac{d \phi}{d r}\right)-h^{\prime \prime}(r) .
$$

At the cathode, $r=a$, using (2), (13), and (15), the initial conditions on $\chi$ are

$$
\chi(r=a)=0=\partial_{r} \chi(r=a) .
$$

To ensure that $\chi$ stays close to zero or assumes negative values for $r \in[a, b]$, the best one may do is look for regimes where $\chi$ (which starts at zero with a zero slope at $r=a$ ) is concave down in the vicinity of the cathode, i.e., choose

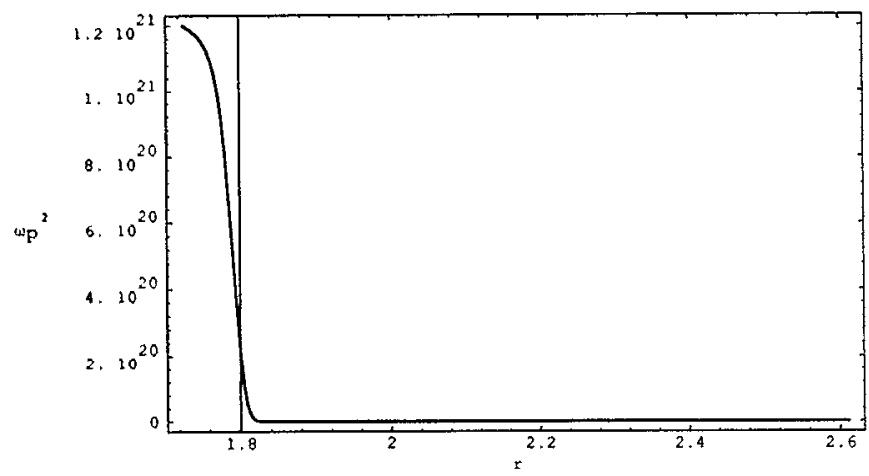

FIG. 3. The density profile in the anode-cathode gap for $\phi$ (anode) $=50 \mathrm{kV}$ in the medium aspect ratio case, where $\rho=0.125$.

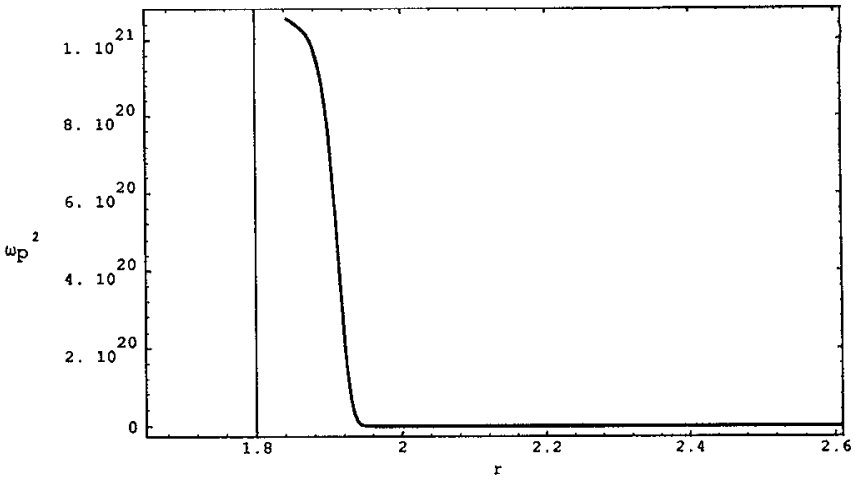

FIG. 4. The same as Fig. 3, but for $\phi($ anode $)=100 \mathrm{kV}$ which is in the lower operating range.

values of $\epsilon$ such that $\partial^{2} \chi / \partial r^{2}(r=a)<0$. Using (46) and (47), $d^{2} \chi / d r^{2}(r=a)=m \beta_{\theta} \beta_{w} \Omega^{2}(1-\epsilon) /\left(\beta_{w}+\beta_{\theta}\right)-h^{\prime \prime}(a)$ $<0$ yields simply

$\epsilon>0$.

Numerical solutions of (46) and (47) were then performed for various positive values of $\epsilon$.

However, it was found that in order to obtain anode voltages, $\phi(b)$, of the order of hundreds of kilovolts typical of such devices, $\epsilon$ would have to assume very small values indeed. For instance, $\phi(b)>100 \mathrm{kV}$ required $\epsilon<O\left(10^{-10}\right)$, and reliable numerical solutions were found to be virtually impossible to obtain, even employing quadprecision and extremely stable composite backward difference schemes. However, the global asymptotic solution of Sec. III had no such limitation, and we turn next to results obtained from this solution.

\section{B. Asymptotic solutions}

Notice that as the anode voltage increases, the electron plasma sheath extends further from the cathode, until it eventually fills the entire cathode-anode gap. This last configuration where the sheath hits the anode corresponds to a voltage "limit," or the highest operating voltage attainable for the device corresponding to a particular set of parameters.

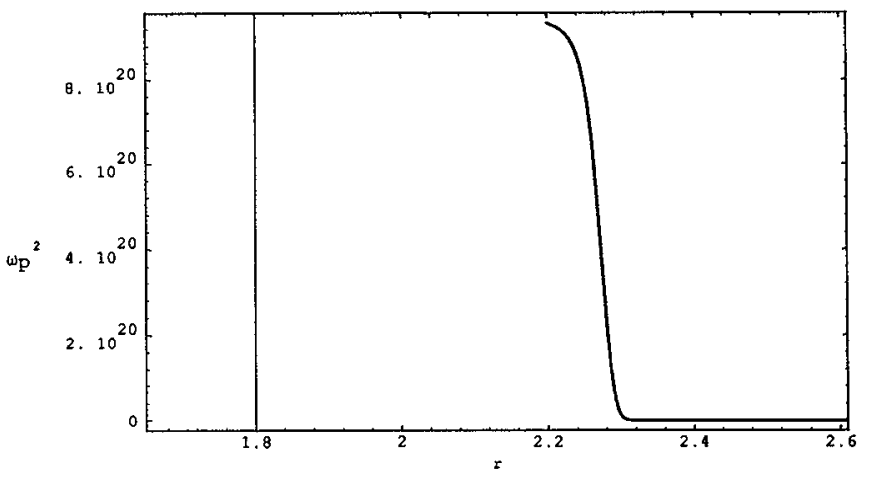

FIG. 5. The same as Fig. 3 for $\phi($ anode $)=200 \mathrm{kV}$. The Hull cutoff voltage is $226 \mathrm{kV}$. 


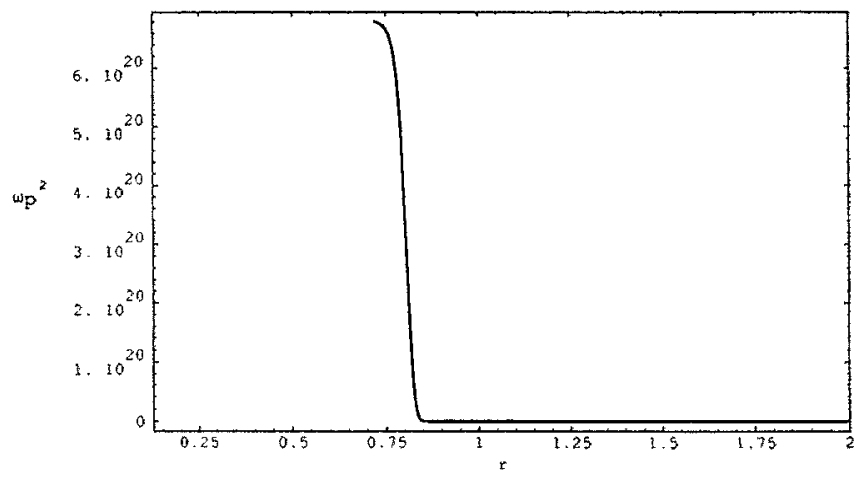

FIG. 6. The density profile for $\phi($ anode $)=100 \mathrm{kV}$ in the high aspect ratio case.

This is also known as the "Hull cutoff voltage," above which the device loses magnetic insulation and goes conducting. A good estimate of the Hull cutoff voltage may be obtained from the asymptotic solution. From Fig. 3 of Ref. 2, the function $J$ of (37) and (38) is positive for all $z$. Enforcing this in (43), yields to $O(1)$ :

$$
\phi<\phi_{\mathrm{Hull}} \equiv \frac{m a^{2} \Omega^{2}}{e}\left(\frac{\left(e^{2 v}-1\right)^{2}}{8\left(e^{2 v}+\rho\right)}+\frac{\ln (H / \mu)}{L^{2}(\rho+1)}\right) .
$$

For our parameters corresponding to the typical medium and large aspect ratio cases, the Hull cutoff voltage is 226.1 and $383.7 \mathrm{kV}$, respectively.

In order to obtain the full numerical solution, (36) [with (30)] was first solved for $\nu(v)$ for each parameter set. From this, one has $\nu\left(v_{f}\right) \equiv \nu_{f}$, where $v_{f}=v_{\text {anode }}=\ln (b / a)$. Next, for the given anode voltage, one may use (43) to obtain the required value of $J\left(z_{f}\right)$ (actually $J-\epsilon J_{10}$; but usually the last term is an insignificant correction), where $z_{f}$ is the value of $z$ at the anode. Now, from the solution of (37) and (38) (or Fig. 3 of Ref. 2), using the required value of $J$, one obtains the required value of $z_{f}$. But $z_{f}$ and $v_{f}$ are related by (32), allowing one to solve for $\epsilon$. Table I shows the required $\epsilon$ values for various anode voltages. At this stage, the reason for the problem being "stiff" or intractable using a pure numerical solution becomes apparent. For instance, for $\phi($ anode $)=100 \mathrm{kV}$, the required value of $\epsilon$ is $O\left(10^{-10}\right)$ and $O\left(10^{-21}\right)$ for the medium and high aspect ratio cases. For the high aspect ratio case, $\epsilon=O\left(10^{-32}\right)$ and $O\left(10^{-45}\right)$ for $\phi($ anode $)=250$ and $350 \mathrm{kV}$, respectively. Thus there is no way that one could numerically calculate the required terms in (30) to a sufficient accuracy. Other methods havc to be used.

Once the required value of $\epsilon$ is known, one may use (45), (30), and the numerical solution of (37) for $J$ to obtain the profile for the density (or the electron plasma frequency, $\left.\omega_{p}^{2}\right)$.

The results are shown in Figs. 2-8. Figure 2 shows the stretched variable $z$ versus the physical variable $v$, as obtained from (32) and (36) for the medium aspect ratio case at $25 \mathrm{kV}$. The very high degree of stretching indicates the difficulty of the problem, as was seen in attempting the pure numerical solution. For higher voltages, the stretching is

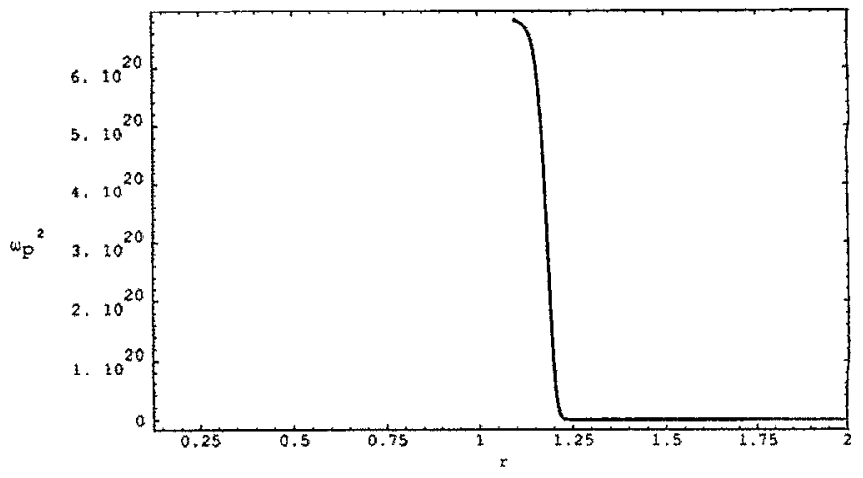

FIG. 7. The same as Fig. 6 for $\phi($ anode $)=250 \mathrm{kV}$

even worse. Figures 3-5 show that density profiles in the anode-cathode gap for the medium aspect ratio case with $\phi$ (anode) $=50,100$, and $200 \mathrm{kV}$, respectively (the Hull cutoff voltage limit is about $226 \mathrm{kV}$ ). Note the widening of the plasma sheath as the voltage increases. Figures $6-8$ show the density profile in the gap for the high aspect ratio case with $\phi($ anode $)=100,250$, and $350 \mathrm{kV}$, respectively (the Hull cutoff voltage is about $383 \mathrm{kV}$ ).

\section{Particle orbits}

To analyze the stability of the particle orbits, we perturb Eqs. (6). One finds that variations in the radial coordinate will evolve as

$$
\delta \ddot{r}+\Delta^{2} \delta r=\left(2 / r^{3}\right) p_{\theta} \delta p_{\theta},
$$

where $\delta p_{\theta}$ is the perturbed angular momentum and

$$
\Delta^{2}=\Omega^{2}-\omega_{p}^{2}-(2 / r)(e / m) \partial_{r} \phi .
$$

The key feature here is that inside the sheath, $\Delta^{2}$ becomes very small (but still positive), allowing large variations in the particle's orbit due to small perturbations. ${ }^{10}$

From (44) and (45), (51) becomes

$$
\begin{aligned}
\frac{\Delta^{2}}{\Omega^{2}}= & 1-\frac{e^{-v}\left(e^{2 v}-1\right)\left(e^{2 v}+1+2 \rho\right)}{2\left(e^{2 v}-1\right)^{2}}-\frac{\mathrm{He}^{-J}}{1+\rho} \\
& +\frac{2 \nu_{v} e^{-2 v}}{L(1+\rho)} J^{\prime} \epsilon \sinh (L \nu)+O\left(\epsilon, 1 / L^{2}\right)
\end{aligned}
$$

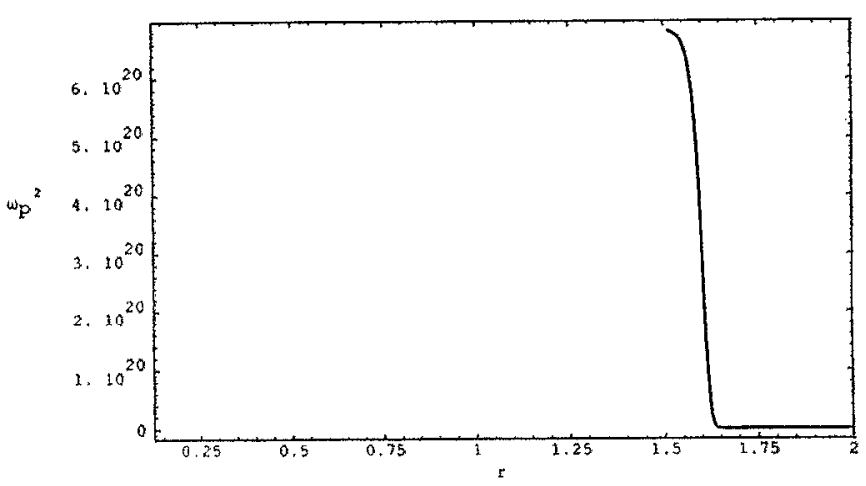

FIG. 8. The same as Fig. 6 for $\phi($ anode $)=350 \mathrm{kV}$. 
which always remains positive definite in lowest order. The second term on the left-hand side is bounded by $1 / 2$, as is also the third term, while the fourth term is obviously positive. Thus depending on the values of $\rho$ and $v$, one could have extremely small values of $\Delta^{2} / \Omega^{2}$, but never zero or negative. In terms of particle orbits, this means that wherever $\Delta^{2} / \Omega^{2} \ll 1$, the orbits are on the verge of an instability, ${ }^{10}$ and that small perturbations to either the angular momentum or the radial position can give large excursions.

\section{ACKNOWLEDGMENTS}

This research was supported in part by the Office of Naval Research and the Air Force Office of Scientific Research.
${ }^{1}$ M. P. Desjarlais and R. N. Sudan, Phys. Fluids 29, 1746 (1986).

${ }^{2}$ D. J. Kaup and G. E. Thomas, Phys. Fluids 31, 2362 (1988).

${ }^{3}$ R. C. Davidson, K. T. Tsang, and J. A. Swegle, Phys. Fluids 27, 2332 (1984).

${ }^{4}$ R. C. Davidson, K. T. Tsang, and H. S. Uhm, Phys. Fluids 31, 1727 (1988).

${ }^{5}$ R. C. Davidson and K. T. Tsang, Phys. Fluids 28, 1169 (1985).

${ }^{6}$ G. E. Thomas, W. M. Bollen, D. J. Kaup, B. Goplen, and L. Ludeking, in Technical Digest-International Conference on Electron Devices, Washington, D.C., 1985 (Institute of Electrical and Electronic Engineers, New York, 1985), pp. 180-183.

${ }^{7}$ R. C. Davidson, Phys. Fluids 27, 1804 (1984).

${ }^{8}$ T. A. Treado, R. A. Bolton, T. A. Hansen, P. D. Brown, and J. D. Barry, IEEE Trans. Plasma Sci. 20, 351 (1992).

${ }^{9}$ S. N. Antani, G. E. Thomas, and D. J. Kaup, J. Plasma Phys. 44, 517 (1990).

${ }^{10}$ D. J. Kaup and G. E. Thomas, Phys. Fluids B 4, 2640 (1992). 